\title{
Variaciones temporales de parámetros físicos, químicos y biológicos de un lago de altura: el caso del lago Chungará
}

\author{
Temporal variations of physical, chemical and biological parameters of a high altitude \\ lake: the case of Chungara lake
}

CRISTINA DORADOR, RODRIGO PARDO \& IRMA VILA ${ }^{1}$

${ }^{1}$ Departamento de Ciencias Ecológicas, Facultad de Ciencias, Universidad de Chile, Las Palmeras 3425, Santiago, Chile; e-mail: limnolog@uchile.cl

\begin{abstract}
RESUMEN
El lago Chungará, es el más austral y de mayor altura de los lagos altoandinos, está ubicado a $4.520 \mathrm{~m}$ de altitud en el altiplano chileno $\left(18^{\circ} 14^{\prime} \mathrm{S}, 6^{\circ} 09^{\prime} \mathrm{O}\right)$; es un lago polimíctico-frío, con origen tectónico- volcánico y $34 \mathrm{~m}$ de profundidad máxima. Presenta variaciones reducidas de temperatura entre profundidades y estaciones del año (4 a 11 ${ }^{\circ} \mathrm{C}$ ), el promedio de temperatura anual se ha incrementado en $1,2^{\circ} \mathrm{C}$ entre los períodos $1986-1987$ y $1998-1999$. Entre los mismos períodos se observó una disminución significativa en el volumen del lago $(-6,58 \%)$ y consecuentemente en la profundidad máxima. Conjuntamente la salinidad aumentó significativamente con valores máximos de 1.464 $\mu$ mhos y $2.650 \mu$ mhos para cada período. Estos cambios pueden ser las causas de la modificación en composición y abundancia del fitoplancton, asociadas, además, a la disminución significativa de la clorofila a $(\mathrm{P}<0,0054)$.
\end{abstract}

Palabras clave: volumen, temperatura, salinidad, fitoplancton.

\begin{abstract}
The Chungará lake ( $\left.18^{\circ} 14^{\prime} \mathrm{S}, 6^{\circ} 09^{\prime} \mathrm{W}\right)$, the most austral and highest among the intertropical andean lakes, is located at $4,520 \mathrm{~m}$ of altitude. It is a polymictic system of tectonic-volcanic origin with a maximum depth of $34 \mathrm{~m}$. It has a narrow temperature range in depth and seasonally $\left(4-11^{\circ} \mathrm{C}\right)$, and which mean annual values show an increment in 1.2 ${ }^{\circ} \mathrm{C}$ between 1986-1987 and 1998-1999 periods. The system total volume has reduced significatively (-6.58\%) during both periods of study and consequently in the maximum depth. Salinity has increased significatively, presenting maximum values of $1,464 \mu$ mhos and $2,650 \mu$ mhos, respectively. These changes would be modifying the phytoplankton composition and abundance and are also probably associated to the significative chlorophyll a decrease between these two periods $(\mathrm{P}<0.0054)$.
\end{abstract}

Key words: volume, temperature, salinity, phytoplankton.

\section{INTRODUCCIÓN}

Los lagos intertropicales altoandinos han sido caracterizados por poseer salinidad alta, baja variación térmica estacional en la columna fitoplanctónica estaría limitada por nitrógeno, lo cual ha sido demostrado experimentalmente por Vincent et al. (1984).

Lago Chungará, es el más austral y de mayor altura de los lagos altoandinos, se encuentra ubicado a $4.520 \mathrm{~m}$ de altitud en el altiplano chileno (18 $14^{\prime}$ ' S, 6909' O). Es un lago polimíctico-frío, con origen tectónico-volcánico y como sistema endorreico de esta zona, presenta alto contenido salino, con profundidades máximas que han variado históricamente entre 34 y $32 \mathrm{~m}$ (Villwock et al. 1985, Mühlhauser et al. 1995). Estas caracte- rísticas de lago tropical de altura y la falta de intervención humana, lo señalan como un sistema importante de estudio de los procesos que relacionen el clima, la geología y la calidad química del agua con las comunidades fitoplanctónicas, especialmente en sistemas donde los niveles de nutrientes no corresponden con la cantidad de clorofila a estimadas para el lago.

En este contexto el régimen pluvial torrencial en cortos períodos de tiempo y con arrastre de materiales que incorporarían sales minerales al sistema, se acentuarían durante los eventos cíclicos generados por el incremento de precipitaciones debido al fenómeno de El Niño (Aceituno 1997). El régimen pluvial tiene fuerte variabilidad interanual y ha sido descrito que durante la fase negativa de la Oscilación del Sur, la precipi- 
tación en el altiplano suele ser deficitaria y específicamente la cuenca del Lago Chungará presenta un balance hídrico negativo durante los últimos años (Aceituno 1997, Salazar 1997). A pesar que los lagos intertropicales se señalan como estables, la salinidad y la composición del fitoplancton del lago Chungará presentan variaciones significativas durante los últimos 13 años. Estos cambios estarían asociados a las variaciones cíclicas interanuales de la precipitación, las cuales a su vez modificarían la calidad física y química del agua.

\section{MATERIALES Y MÉTODOS}

El lago Chungará (18²14' S, 6909' O) se ubica en el Parque Nacional Lauca, Reserva de la Biósfera de UNESCO. La hoya hidrográfica alcanza los $260 \mathrm{~km}^{2}$ (Fig. 1), con un volumen promedio de $400 \mathrm{Hm}^{3}$ y una profundidad máxima de $34 \mathrm{~m}$ (Mühlhauser et al. 1995). El tributario principal es el río Chungará con un flujo entre 300-500 L s'${ }^{-1}$.

Con el objeto de analizar la calidad física y química del agua y su relación con la cantidad de clorofila a en el lago, se realizaron cuatro muestreos durante los meses de agosto, enero, mayo y septiembre, entre 1998 y 1999 en una estación fija de la zona de profundidad máxima, la cual alcanzó a los $32 \mathrm{~m}$. Se obtuvieron muestras de agua con una botella Van Dorn a 0,15 y $30 \mathrm{~m}$ de profundidad, midiéndose: temperatura con un termómetro digital Hanna Instruments de 0,1 de precisión, conductividad con un conductivímetro VWR y pH con un electrodo Hanna Instruments. Se determinó oxígeno disuelto según el método de Winkler (APHA 1998) y el porcentaje de saturación se calculó corrigiendo por altura. Para estimar cantidad de clorofila a, la muestra de agua fue filtrada y extraída (en frío con acetona $90 \%$ ) de acuerdo con Cabrera (1984). Para la determinación de la concentración $\mathrm{P}_{-} \mathrm{PO}_{4}$ se utilizó el método de Zahradnik (1981). La determinación de $\mathrm{N}-\mathrm{NO}_{3}$ se hizo de acuerdo a Mühlhauser et al. (1987). La información obtenida se comparó con la de Mladinic et al. (1987), la cual fue medida con metodología afín, mediante un ANDEVA de dos factores y de un factor (Zar 1996).

Para evaluar cualitativamente y cuantificar el fitoplancton se tomaron muestras de agua cada $1 \mathrm{~m}$ entre superficie y $\operatorname{los} 7 \mathrm{~m}$ de profundidad. Las muestras fueron fijadas en Lugol y analizadas de acuerdo con el método de Utermöhl en un micros-

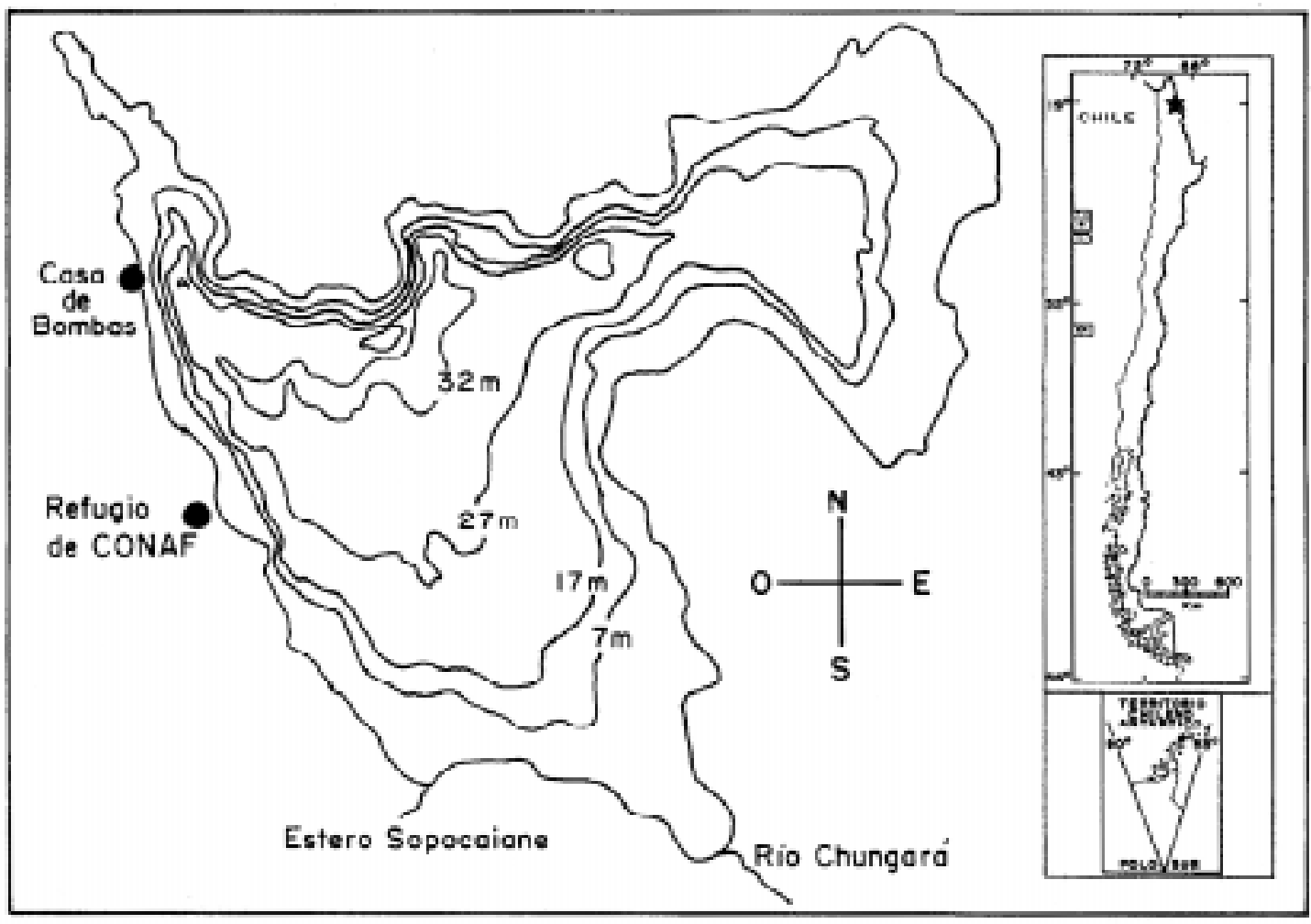

Fig 1: Ubicación geográfica y batimetría del lago Chungará (modificado de Villwock et al. 1985). Geographic location and bathymetric map of Lake Chungará (modified from Villwock et al. 1985). 
copio invertido modelo Olympus CK2. La abundancia se evaluó en unidades estándar/litro, cada unidad se calibró a $400 \mu^{2}$, (Sournia 1978). La identificación taxonómica se realizó usando las descripciones de Liberman \& Miranda (1987) y Parra \& Bicudo (1995). Los resultados de composición y abundancia para el período 1998-1999 se compararon con antecedentes cualitativos y cuantitativos de fitoplancton provenientes de la misma estación de muestreo y obtenidas con la misma metodología en superficie, durante abril de 1980.
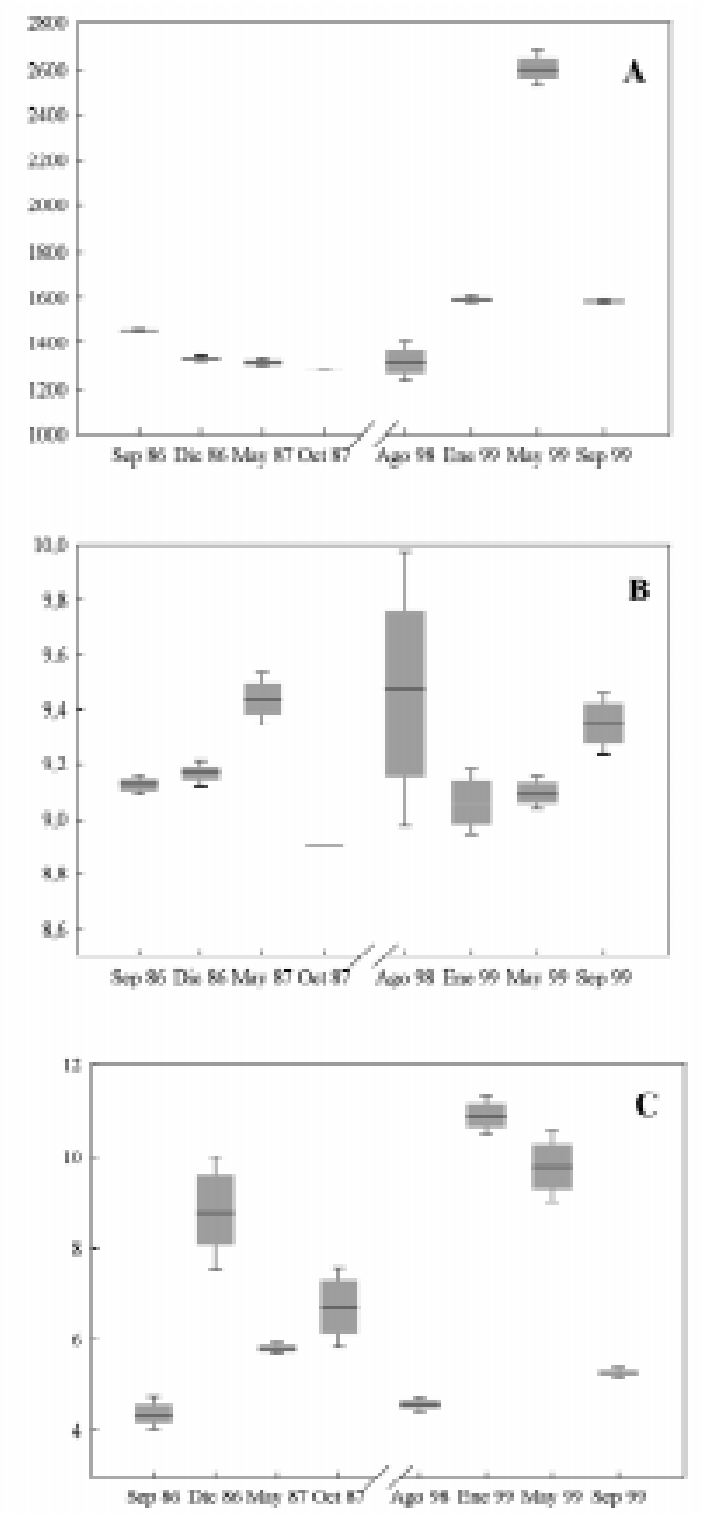

\section{RESULTADOS}

Variación de la calidad química entre meses en la columna de agua

La conductividad (Fig. 2A y 3), presentó el valor más bajo en el período 1986-1987 y correspondió a $1.464 \mu$ mhos en septiembre 1986 a $15 \mathrm{~m}$, mientras que en el período 1998-1999 la conductividad fue de $2.650 \mu$ mhos en mayo 1999 a $15 \mathrm{~m}$ de profundidad. La temperatura varió entre $\operatorname{los} 4,1^{\circ} \mathrm{C}$
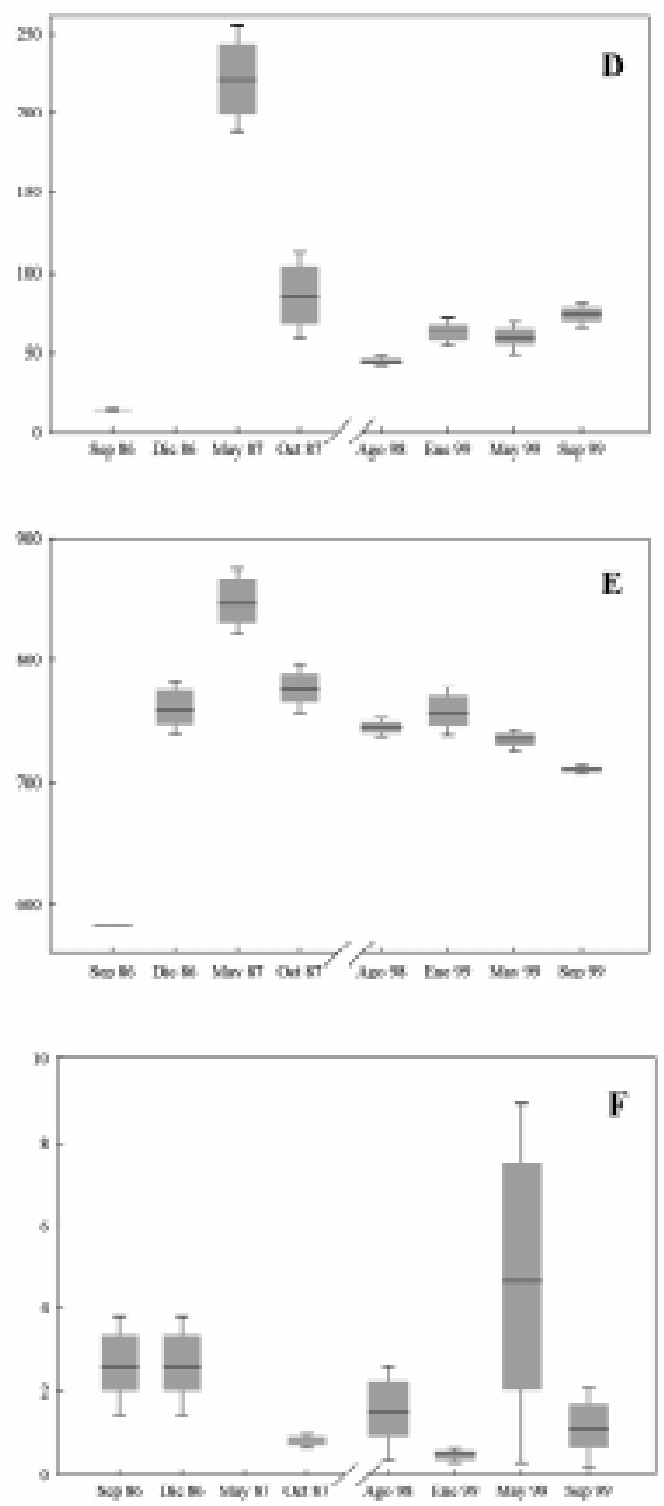

Fig 2: Variables físicas: (A) conductividad, (B) $\mathrm{pH}$, (C) temperatura. Variables químicas: (D) $\mathrm{N}^{-\mathrm{NO}}{ }_{3}$, (E) $\mathrm{P}_{-} \mathrm{PO}_{4}$ y (F) Clorofila a. Promedios de las tres profundidades estudiadas, las cajas el error estándar y las líneas marcan 1,96 veces el error estándar.

Physical variables: (A) conductivity, (B) $\mathrm{pH},(\mathrm{C})$ temperature. Chemical variables: (D) N-NO,$(E) \mathrm{P}_{3} \mathrm{PO}_{4}$ and (F) Chlorophyll a. Average values for three depth analyzed, boxes the standard error and lines mark 1.96 times the standard error. 


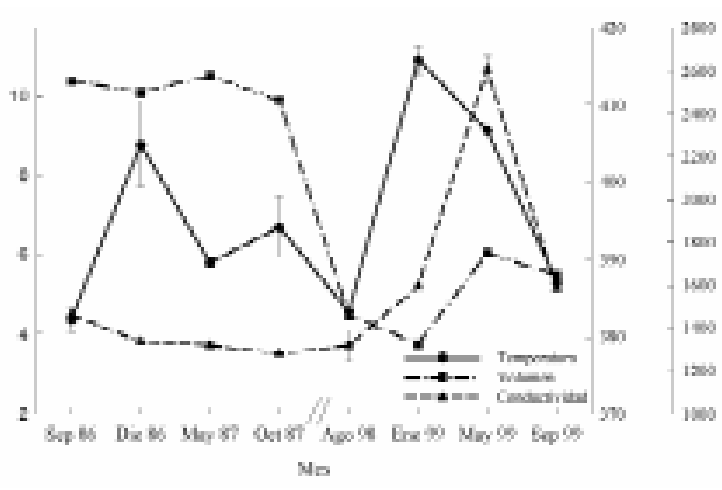

Fig 3: Temperatura $\left({ }^{\circ} \mathrm{C}\right)$, volumen $\left(\mathrm{Hm}^{3}\right)$ y conductividad ( $\mu$ mhos) del lago Chungará durante los períodos de estudio. Las líneas segmentadas y punteadas representan la tendencia de estas variables en el tiempo.

Lake Chungará temperature $\left({ }^{\circ} \mathrm{C}\right)$, volume $\left(\mathrm{Hm}^{3}\right)$ and conductivity ( $\mu$ mhos) during the study periods. The dash and dotted line represents the tendency of these variables in time.

en septiembre de 1986 a $34 \mathrm{~m}$ de profundidad y $11,2{ }^{\circ} \mathrm{C}$ en enero de 1999 en la superficie (Fig. 2C y 3). El pH (Fig. 3B) se mantuvo básico en ambos períodos y sus valores máximos fluctuaron entre 9,5 en mayo 1987 a $15 \mathrm{~m}$ de profundidad y 9,8 en agosto 1998 a $32 \mathrm{~m}$ de profundidad.

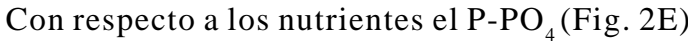
presentó el máximo en mayo de 1987 con $872 \mu \mathrm{g}$ $\mathrm{L}^{-1}$ y mínimo en septiembre 1986 con $584 \mu \mathrm{g} \mathrm{L}^{-1}$. El valor máximo de $\mathrm{N}-\mathrm{NO}_{3}$ (Fig. 2D) se observó en superficie durante mayo 1987 y alcanzó los $250 \mu \mathrm{g} \mathrm{L}^{-1}$, el mínimo se presentó en septiembre de 1986 con $14 \mu \mathrm{g} \mathrm{L}^{-1}$ a $15 \mathrm{~m}$.

La clorofila a (Fig. 2F) fluctuó entre los 0,34 $\mathrm{mg} \mathrm{L}^{-1}$ a $15 \mathrm{~m}$ en enero de 1999 y $8,74 \mathrm{mg} \mathrm{L}^{-1}$ en mayo de 1999 a la misma profundidad. La concentración de oxígeno disuelto presentó fluctuaciones menores a profundidades diferentes, con la excepción de enero de 1999 (8,77 y 4,19 $\mathrm{mg} \mathrm{L}^{-1}$; 126,35 y $63,89 \%$ de saturación respectivamente). Los valores fluctuaron en superficie entre 5,13 $\mathrm{mg} \mathrm{L}^{-1}$ (76,2 \% de saturación) en diciembre de 1986 y 9,02 (114,42\% saturación) en agosto de 1998. A $32 \mathrm{~m}$ fluctuaron entre $4,93 \mathrm{mg} \mathrm{L}^{-1}$ (70,92 \% saturación) en diciembre de 1986 y $8,9 \mathrm{mg} \mathrm{L}^{-1}$ (112,14\% saturación) en agosto de 1998.

La temperatura, conductividad, nitratos, fosfatos y clorofila a, durante los periodos 19861987 y 1998-1999 presentaron diferencias significativas entre los meses estudiados (Tabla 1). Por otra parte, se encontró que la clorofila a, fosfatos, temperatura y conductividad no diferían entre profundidades durante ambos períodos, sólo los nitratos presentaron diferencias en la columna de agua, debido posiblemente a la influencia de los meses de mayo y octubre de 1987, los que además de ser los que poseen valores más altos, son los que presentaron mayores diferencias entre profundidades (Fig. 2D). Apoyados en el hecho de que los meses estudiados presentan una marcada homogeneidad en la columna de agua se decidió tomar, para los análisis siguientes las tres profundidades como réplicas de las variables en cada mes.

\section{Variabilidad entre años}

El volumen del lago disminuyó significativamente durante los últimos 13 años (ANDEVA dos factores sin réplica, $\mathrm{P}>0,0017)$, mostrando un descenso en un $6,58 \%$ durante los dos períodos de estudio que correspondieron a los años 19861987 y $1998-1999$, con promedios de $412,32 \pm$ $0,69 \mathrm{Hm}^{3}$ y $385,17 \pm 2,62 \mathrm{Hm}^{3}$. El volumen muestra estabilidad anual, no presentando diferencias entre estaciones del año (ANDEVA dos factores sin réplica, $\mathrm{P}>0,425)$ (Fig. 3).

Al comparar, mediante ANDEVAS de dos factores con réplica entre años y meses (Zar 1996) el período de estudio 1998-1999 con el estudio efectuado entre los años 1986 y 1987 se encontró que los factores analizados presentaron variaciones altamente significativas entre estaciones del año (Tabla 2) y que tanto la temperatura, que aumentó de $6,41 \pm 1,76{ }^{\circ} \mathrm{C}$ a $7,64 \pm 2,89{ }^{\circ} \mathrm{C}$ como la conductividad, cuyos promedios y sus respectivas desviaciones estándar varían desde 1.350,3 \pm 20,33 a $1.775,8 \pm 148,85 \mu$ mhos, presentaron diferencias significativas entre períodos. Por el contrario, la clorofila a disminuyó entre los dos períodos de estudio (2,0 y $1,9 \mathrm{mg} \mathrm{L}^{-1}$, Tabla 2$)$, cabe mencionar que para este último análisis no se incluyó la estación de otoño debido a que no

TABLA 1

Análisis de varianza de dos factores sin réplica entre años y meses

Replicate two factors ANOVA between years and months

Valores de probabilidad luego de realizar un ANDEVA de dos factores sin réplica

\begin{tabular}{lcc} 
Factor & \multicolumn{1}{c}{ Meses } & Profundidad \\
\hline Clorofila a & $<0,0081^{*}$ & $>0,717$ \\
Fosfatos & $<<0,0001^{* * *}$ & $>0,154$ \\
Nitratos & $<<0,0001^{* * *}$ & $<0,033^{*}$ \\
Temperatura & $<<0,0001^{* * *}$ & $>0,061$ \\
Conductividad & $<<0,0001^{* * *}$ & $>0,249$ \\
\hline
\end{tabular}


TABLA 2

Análisis de varianza de dos factores (Período, Estación) para las variables físicas, químicas y clorofila a del Lago Chungará

Two-way analysis of variance (period, season) for physical, chemical and chlorophyll a variables at Chungará Lake

\begin{tabular}{|c|c|c|c|c|c|c|c|}
\hline Variable & Fuente de variación & $\begin{array}{c}\text { Grados de } \\
\text { libertad } \\
\text { del error }\end{array}$ & $\begin{array}{c}\text { Suma de } \\
\text { cuadrados } \\
\text { del error }\end{array}$ & $\begin{array}{c}\text { Grados de } \\
\text { libertad } \\
\text { del factor }\end{array}$ & $\begin{array}{c}\text { Suma de } \\
\text { cuadrados } \\
\text { del factor }\end{array}$ & $\begin{array}{l}\text { Valor } \\
\text { de F }\end{array}$ & $\begin{array}{l}\text { Valor } \\
\text { de P }\end{array}$ \\
\hline \multirow[t]{3}{*}{ Temperatura } & Período & 1 & 9,06 & 16 & 0,3063 & 29,59 & 0,0001 \\
\hline & Estación & 3 & 32,14 & 16 & 0,3063 & 104,91 & $<<0,0001$ \\
\hline & Período x estación & 3 & 8,18 & 16 & 0,3063 & 26,72 & $<<0,0001$ \\
\hline \multirow[t]{3}{*}{$\mathrm{PH}$} & Período & 1 & 0,04 & 16 & 0,0272 & 1,71 & 0,2083 \\
\hline & Estación & 3 & 0,05 & 16 & 0,0272 & 2,01 & 0,1536 \\
\hline & Periodo x estación & 3 & 0,21 & 16 & 0,0272 & 7,74 & 0,0020 \\
\hline \multirow[t]{3}{*}{ Conductividad } & Período & 1 & 7,96 & 16 & 0,0910 & 87,52 & $<<0,0001$ \\
\hline & Estación & 3 & 0,92 & 16 & 0,0910 & 10,09 & 0,0006 \\
\hline & Período x estación & 3 & 3,74 & 16 & 0,0910 & 41,11 & $<<0,0001$ \\
\hline \multirow{3}{*}{ P-PO 4} & Período & 1 & 201,84 & 16 & 210,16 & 0,96 & 0,3417 \\
\hline & Estación & 3 & $17.409,05$ & 16 & 210,16 & 82,83 & $<<0,0001$ \\
\hline & Período x estación & 3 & $21.633,15$ & 16 & 210,16 & 102,93 & $<<0,0001$ \\
\hline \multirow[t]{3}{*}{$\mathrm{N}-\mathrm{NO} 3$} & Período & 1 & 0,04 & 12 & 0,0214 & 1,72 & 0,2144 \\
\hline & Estación & 2 & 3,71 & 12 & 0,0214 & 173,36 & $<<0,0001$ \\
\hline & Período x estación & 2 & 2,33 & 12 & 0,0214 & 108,63 & $<<0,0001$ \\
\hline \multirow[t]{3}{*}{ Clorofila } & Período & 1 & 2,24 & 12 & 0,1954 & 11,47 & 0,0054 \\
\hline & Estación & 2 & 0,82 & 12 & 0,1954 & 4,20 & 0,0414 \\
\hline & Período x estación & 2 & 1,29 & 12 & 0,1954 & 6,65 & 0,0114 \\
\hline
\end{tabular}

Período: años 1986-1987 / años 1998-1999

Period: years 1986-1987 / years 1998-1999

Estación: estación del año

Season: year season

existían observaciones anteriores para este período; para precisar el nivel de resolución del análisis, se realizó un ANDEVA de un factor (Zar 1996) para la clorofila a, tomando todos los meses estudiados (Fig. 2F) presentando diferencias entre meses y observándose una división de los meses según sus cantidades. Los meses de octubre de 1987 y enero de 1999 presentaron los valores más bajos diferenciándose significativamente del mes de mayo de 1999 que mostró la mayor cantidad (prueba de Tukey; P < 0,04 y P < 0,004, respectivamente), además se observó que enero de 1999 se diferenció de septiembre de 1986 y diciembre de 1986 (prueba de Tukey; P< 0,02 para ambos). No se encontraron diferencias entre períodos en los valores de $\mathrm{pH}$, el cual aumentó levemente de 9,15 $\pm 0,2$ a 9,24 $\pm 0,26$, del fósforo que varió de 742,58 $\pm 29,63$ a 736,78 \pm $5,89 \mu \mathrm{g} \mathrm{L}^{-1}$ ni del nitrógeno que presentó promedios de $107 \pm 31,03 \mu \mathrm{g} \mathrm{L}^{-1}$ y $60,25 \pm 3,43 \mu \mathrm{g} \mathrm{L}^{-1}$ para cada período (Tabla 1).

La Tabla 3 detalla la abundancia relativa de las microalgas durante abril de 1980, mes durante el cual el grupo más abundante estuvo representado por Clorophyceae y Bacillariophyta siendo las especies más representativas Botryococcus braunii Kutzing, Stephanodiscus astraea (Kutz.) Grunow y Lagynion sp. Durante el período 19981999 los grupos más abundantes fueron Chlorophyceae y Bacillariophyta con las especies más representadas por Coelastrum microporum Naegeli, Botryococcus braunii Kutzing y Stephanodiscus astraea (Kutz.) Grunow. Bacillariophyta fue el grupo más abundante durante el mes agosto 1998 (79,8\%), uno de los meses más fríos, mientras que Clorophyceae presenta las mayores abundancias durante los meses más cálidos: abril de 1980, enero de 1999, mayo de 1999 y septiembre de 1999.

\section{DISCUSIÓN}

El lago Chungará es un sistema polimíctico frío con amplitud de variaciones de temperatura reducida estacionalmente y en profundidad, típica de las regiones tropicales a semejanza del lago Menor y de las bahías poco profundas en la cuenca del lago Titicaca (Iltis et al. 1991, Aceituno 1997, Salazar 1997), esta estabilidad anual disminuye 
la variación de los factores físicos responsables de la dinámica de la composición y abundancia de las microalgas, asociándose principalmente a dos períodos: de lluvias y de período seco.

El aumento de la temperatura del lago entre los años 1986 y 1999 podría deberse a la disminución en el volumen del lago, el cual ha perdido aproximadamente un $7 \%$ durante el mismo período. El mayor contenido salino, que se observa en los valores de conductividad del agua se explica por los procesos de erosión que ocurren en la cuenca como producto de avenamiento en los terrenos aledaños, hecho especialmente notorio durante el período estival y procesos de concentración salina en los períodos de menor pluviosidad debido a la disminución del volumen del lago.

Los procesos biogeoquímicos de los lagos altoandinos se ven influenciados por la geomorfología de la cuenca, flujo de irradiación solar importante, bajas temperaturas y deficiencia de oxígeno, variables que conjuntamente con la disponibilidad de nutrientes regulan la produc-

TABLA 3

Abundancia relativa de microalgas (\%) durante abril de 1980, agosto de 1998, enero, mayo y septiembre de 1999

Phytoplankton relative abundance (\%) during April 1980, August 1998, Juanuary, May and September 1999

\begin{tabular}{|c|c|c|c|c|c|}
\hline Especie & $\begin{array}{c}\text { Abril } \\
1980\end{array}$ & $\begin{array}{c}\text { Agosto } \\
1998\end{array}$ & $\begin{array}{c}\text { Enero } \\
1999\end{array}$ & $\begin{array}{c}\text { Mayo } \\
1999\end{array}$ & $\begin{array}{c}\text { Septiembre } \\
1999\end{array}$ \\
\hline Botryococcus braunii & 90,57 & 0,16 & 6,15 & 43,63 & 1,96 \\
\hline Stephanodiscus astraea & 3,95 & 67,56 & 4,98 & 29,66 & 10,06 \\
\hline Coelastrum microporum & 0,00 & 5,96 & 19,32 & 16,62 & 41,23 \\
\hline Cyclotella stelligera & 0,00 & 7,04 & 12,09 & 3,34 & 8,32 \\
\hline Oocystis sp. & 0,14 & 0,83 & 15,66 & 0,19 & 3,75 \\
\hline Closterium acutum & 0,02 & 7,34 & 0,05 & 2,03 & 8,69 \\
\hline Schroederia setigera & 0,30 & 0,35 & 13,91 & 0,58 & 2,34 \\
\hline Mougeotia sp. & 0,00 & 0,07 & 0,00 & 0,00 & 13,51 \\
\hline Aulacoseira granulata & 0,00 & 0,91 & 6,67 & 0,06 & 2,11 \\
\hline Sphaerocystis schroeteri & 1,89 & 1,87 & 2,26 & 0,42 & 0,35 \\
\hline Gloeocapsa sp. & 0,00 & 0,00 & 4,86 & 0,03 & 1,59 \\
\hline Cocconeis placentula & 0,34 & 1,91 & 0,98 & 0,45 & 2,23 \\
\hline Oscillatoria $\mathrm{sp}$ & 0,00 & 0,61 & 3,30 & 0,05 & 0,97 \\
\hline Microcystis aeruginosa & 0,00 & 0,00 & 4,64 & 0,00 & 0,00 \\
\hline Anabaena sp. & 0,00 & 0,15 & 2,51 & 0,00 & 0,98 \\
\hline Cryptomonas sp. & 0,00 & 1,62 & 0,00 & 1,30 & 0,00 \\
\hline Chroococcus sp. & 0,00 & 0,00 & 2,04 & 0,33 & 0,22 \\
\hline Pediastrum duplex & 0,00 & 2,19 & 0,00 & 0,00 & 0,00 \\
\hline Lagynion sp. & 1,47 & 0,00 & 0,00 & 0,00 & 0,00 \\
\hline Nephroclamys subsolitaria & 1,02 & 0,00 & 0,03 & 0,12 & 0,00 \\
\hline Navicula sp. & 0,00 & 0,34 & 0,19 & 0,03 & 0,42 \\
\hline Fragilaria crotonensis & 0,00 & 0,00 & 0,00 & 0,19 & 0,62 \\
\hline Dictiosphaerium pulchellum & 0,00 & 0,00 & 0,09 & 0,26 & 0,08 \\
\hline Scenedesmus ecornis & 0,00 & 0,05 & 0,00 & 0,00 & 0,35 \\
\hline Merismopedia sp. & 0,00 & 0,00 & 0,00 & 0,38 & 0,00 \\
\hline Lyngbia sp. & 0,00 & 0,35 & 0,00 & 0,00 & 0,00 \\
\hline Ankistrodesmus falcatus & 0,00 & 0,33 & 0,00 & 0,00 & 0,00 \\
\hline Epithenia sp. & 0,00 & 0,07 & 0,20 & 0,00 & 0,04 \\
\hline Synedra ulna & 0,30 & 0,00 & 0,00 & 0,00 & 0,00 \\
\hline Elakatothrix viridis & 0,00 & 0,03 & 0,00 & 0,00 & 0,20 \\
\hline Coscinodiscus sp. & 0,00 & 0,03 & 0,00 & 0,15 & 0,00 \\
\hline Gleocystis sp. & 0,00 & 0,00 & 0,00 & 0,11 & 0,00 \\
\hline Staurastrum gracile & 0,00 & 0,10 & 0,00 & 0,00 & 0,00 \\
\hline Rhoiscosphenia sp. & 0,00 & 0,07 & 0,00 & 0,00 & 0,00 \\
\hline Tetraedron minimum & 0,00 & 0,03 & 0,01 & 0,03 & 0,00 \\
\hline Cosmarium sp. & 0,00 & 0,00 & 0,05 & 0,01 & 0,00 \\
\hline Dunaliella sp. & 0,00 & 0,03 & 0,00 & 0,00 & 0,00 \\
\hline Desmidium swartzii & 0,00 & 0,00 & 0,00 & 0,03 & 0,00 \\
\hline Crucigenia sp. & 0,00 & 0,00 & 0,01 & 0,00 & 0,00 \\
\hline
\end{tabular}


ción biológica (Vincent et al. 1986). En el caso particular del lago Chungará, se observó que los valores de $\mathrm{P}-\mathrm{PO}_{4}$ y N-NO 3 no presentaron diferencias entre períodos, por lo que los cambios de la productividad del sistema reflejado en la clorofila a, se deberían a cambios en otros factores tales como el aumento de la conductividad y/o temperatura. Esta última, se ha considerado como el factor modelador de mayor relevancia en cuanto a la productividad y estructura fitoplanctónica (Reynolds et al. 2000).

Los valores de $\mathrm{P}-\mathrm{PO}_{4}$ corresponden a valores de sistemas eutróficos (Ryding \& Rast 1992). Wurtsbaugh et al. (1991) e Iltis (1991) discuten la generalización aceptada del fósforo como elemento limitante de la producción fitoplanctónica, hecho que no se produce en los lagos intertropicales. A semejanza de lo que ocurre con otros lagos tropicales y con los lagos nordpatagónicos (Weinstein 2002), el lago Chungará mostraría también limitación por nitrógeno. Debido a las características medioambientales de este lago de altura es interesante analizar la estructura de la comunidad fitoplanctónica como primera aproximación hacia un estudio detallado de las fluctuaciones que experimentarían estas comunidades frente a efectos antrópicos y/o a las variables hidrológicas del altiplano ya que de acuerdo con Andrew et al. (1989), el zooplancton muestra bajas tasas respiratorias y tiene principalmente distribución superficial, razón por la cual la herviboría no explicaría la reducida biomasa algal.

La riqueza de especies de microalgas es relativamente baja en estos sistemas y más que endemismo, las especies dominantes presentarían adaptaciones a las condiciones ambientales de cada período. El lago Chungará presenta una riqueza de especies menor que el lago Titicaca.

El aumento en un grado de temperatura desde el período 1986-1987 al 1998-1999, podría explicar la disminución en la clorofila a, debido posiblemente a la presencia de especies menos tolerantes a temperaturas mayores, siendo las especies presentes en el lago, más bien, cosmopolitas. Es decir, se estarían flexibilizando los factores limitantes de la producción biológica, lo que ha permitido el aumento de la riqueza de especies.

La información limnológica acerca del lago Chungará es aún fragmentaria y requiere conjuntamente de la comprobación experimental con la obtención de información a largo plazo, conocimiento que permitiría explicar las respuestas de los organismos planctónicos a las condiciones de altura. Sin embargo, las características de estos sistemas permitirían afirmar su fragilidad ante los balances hidrológicos negativos del área y la tendencia del sistema a disminuir su volumen de agua. El lago Chungará podría estar disminuyendo su volumen a niveles críticos dado que por las características de los suelos de la cuenca (Charrier 1997) este podría ser un factor que aceleraría el proceso de salinización del sistema lacustre.

\section{AGRADECIMIENTOS}

Los autores agradecen a la Dirección General de Aguas (DGA), Departamento de Estudios del Ministerio de Obras Públicas por el financiamiento parcial del trabajo en terreno y en especial al Señor Carlos Salazar. Por el apoyo técnico se agradece a Vilma Barrera y Lorena Soto, y finalmente, por los valiosos comentarios a Vivian Montecino y a dos correctores anónimos.

\section{LITERATURA CITADA}

ACEITUNO P (1997) Aspectos generales del clima en el altiplano sudamericano. En: Charrier R, P Aceituno, M Castro, A Llanos \& LA Raggi (eds) El Altiplano: ciencia y conciencia de Los Andes: 63-69. Actas del Segundo Simposio Internacional de Estudios Altiplánicos, Santiago, Chile.

APHA (1999) Standard methods for the examination of water and wastewater. Twentieth edition. Baltimore, Maryland. $1.325 \mathrm{pp}$.

ANDREW T, S CABRERA \& V MONTECINO (1989) Diurnal changes in zooplankton respiration rates and the phytoplankton activity in two Chilean lakes. Hydrobiologia 175: 121-135.

CABRERA S (1984) Estimación de la concentración de clorofila a y feopigmentos: una revisión metodológica. En: Bahamonde N \& S Cabrera (eds) Embalses, fotosíntesis y productividad primaria: 189-200. Alfa-Beta Ediciones, Santiago, Chile.

CHARRIER R (1997) Geología y tectónica del altiplano chileno. En: Charrier R, P Aceituno, M Castro, A Llanos \& LA Raggi (eds) El Altiplano: ciencia y conciencia de Los Andes: 23-31. Actas del Segundo Simposio Internacional de Estudios Altiplánicos, Santiago, Chile.

ILTIS A (1991) Aspecto cuantitativo y poblaciones. En: Dejoux C \& A Iltis (eds) El Lago Titicaca: síntesis del conocimiento limnológico actual: 199-210. Orstom/ Hisbol, La Paz, Bolivia.

ILTIS A, J CARMOUZE \& J LEMOALLE (1991) Características físico-químicas del agua. En: Dejoux C \& A Iltis (eds) El Lago Titicaca: síntesis del conocimiento limnológico actual: 107-113. Orstom/Hisbol, La Paz, Bolivia.

LIBERMAN M \& C MIRANDA (1987) Contribución al conocimiento del fitoplancton del lago Titicaca. Oldepesca, Documento de pesca No. 003, Lima, Perú. $82 \mathrm{pp}$. 
LÖFFLER H (1960) Limnologische Untersuchungen an chilenischen und peruanischen Binnengewässern. Die Physikalisch-chemischen Verhältnisse. Ahile) 20: 8994.

MÜHLHAUSER H, L SOTO \& P ZAHRADNIK (1987) Improvement of the Kjeldahl method for total nitrogen including acid hydrolizable phosphorus determinations in freshwater ecosystems. International Journal of Environmental Analitycal Chemistry 28: 215-226.

MÜHLHAUSER H, N HREPIC, P MLADINIC, V MONTECINO \& S CABRERA (1995) Water quality and limnological features of a high altitude Andean lake, Chungará, in northern Chile. Revista Chilena de Historia Natural 68: 341-349.

PARRA O \& C BICUDO (1995) Introducción a la biología y sistemática de las algas de aguas continentales. Ediciones Universidad de Concepción, Concepción, Chile. 268 pp.

REYNOLDS C, M DOKULIL \& J PADISACK (2000) Understanding the assembly of phytoplankton in relation to the trophic spectrum: where are we now? Hydrobiologia 424: 147-152.

RICHERSON P, P NEALE, W WURTSBAUGH, R ALFARO \& W VINCENT (1986) Patterns of temporal variation in Lake Titicaca, a high altitude tropical lake. 1: background, physical and chemical processes and primary production. Hydrobiologia 138: 205 220.

RICHERSON P, P NEALE, R ALFARO, X LAZZARO, W VINCENT \& W WURTSBAUGH (1991) Producción planctónica primaria y biomasa algal. En: Dejoux C \& A Iltis (eds) El Lago Titicaca: síntesis del conocimiento limnológico actual: 211-229. Orstom/Hisbol, La Paz, Bolivia.

RYDING S-O \& W RAST (1992) El control de la eutrofización en lagos y pantanos. UNESCO, The Partenon Publishing Group, París, Francia. 375 pp.

SALAZAR C (1997) Hidrología del sector altiplánico chileno. En: Charrier R, P Aceituno, M Castro, A Llanos \& LA Raggi (eds) El Altiplano: ciencia y conciencia de Los Andes: 71-77. Actas del Segundo Simposio Internacional de Estudios Altiplánicos, Santiago, Chile.
SOURNIA A (1978) Phytoplankton manual. Monographs on Oceanographic Methodology, 6, UNESCO Publishing, París, Francia. 337 pp.

VILA I \& H MÜLHAUSER (1987) Dinámica de lagos de altura, perspectivas de investigación. Archivos de Biología y Medicina Experimentales (Chile) 20: 95 103.

VILLWOCK W, L KIES, F THIEDIG \& R THOMANN (1985) Geologisch-ökologische Untersuchungen am Lago Chungará / Nord Chile: Zielsetzungen und erste Ergebnisse. IDESIA (Chile) 9: 21-34.

VINCENT W, W WURTSBAUGH, C VINCENT \& P RICHERSON (1984) Seasonal dynamics of nutrient limitation in a tropical high-altitude lake (Lake Titicaca, Perú-Bolivia): application of physiological bioassays. Limnology \& Oceanography 29: 540-552.

VINCENT W, C VINCENT, $M$ DOWNES \& $P$ RICHERSON (1985) Nitrate cycling in lake Titicaca (Perú-Bolivia): the effect of high altitude and tropicality. Freshwater Biology 15: 31-42.

VINCENT W, WURTSBAUGH W, P NEALE, P RICHERSON (1986) Polimixis and algal production:latitudinal effects on the seasonality of photosynthesis. Freshwater Biology 16: 781-803.

WEINSTEIN P (2002) Modelos predictivos de nutrientes y clorofila "a" en lagos chilenos. Tesis de Magister en Ciencias de la Ingeniería, Facultad de Ingeniería, Pontificia Universidad Católica de Chile, Santiago, Chile. 137 pp.

WURTSBAUGH W, W VINCENT, R ALFARO, C VINCENT \& P RICHERSON (1985) Nutrient limitation of algal growth and nitrogen fixation in a tropical alpine lake, Lake Titicaca (Perú/Bolivia). Freshwater Biology 15: 185-195.

WURTSBAUGH W, W VINCENT, C VINCENT, H CARNEY, P RICHERSON, X LAZZARO \& R TAPIA (1991) Nutrientes y su limitación del crecimiento del fitoplancton. En: Dejoux C \& A Iltis (eds) El Lago Titicaca: síntesis del conocimiento limnológico actual: 161-175. Orstom/Hisbol, La Paz, Bolivia.

ZAHRADNIK P (1981) Methods for chemical analysis of inland waters. Lecture notes for the International Graduate Training Course on Limnology. Limnologishes Institut. Österreichische Akademie der Wissenschaften, Viena, Austria, Leaflet 44 pp.

ZAR J (1996) Biostatical analysis. Third edition. PrenticeHall, Englewood Cliffs, New Jersey. 660 pp. 